\title{
Parameters of evaluating oral condition from the viewpoint of demineralization
}

\begin{abstract}
Koji Watanabe ${ }^{1}$, Shigeru Watanabe ${ }^{2 *}$ and Kenshi Maki $^{3}$
One of the major concerns in oral health is tooth demineralization. Enamel demineralization consists of caries and erosion. Caries is determined as demineralization due to bacterially derived acid production, and erosion is determined as demineralization due to nonbacterially derived acid such as frequent intake of acid food or drink. In this section, two topics of evaluating oral condition from the viewpoint of demineralization are shown; one is the relationship between caries experience and the concentration of some trace elements in their resting mixed saliva, and the other is evaluation of enamel mineral density using micro-CT.
\end{abstract}

${ }^{1}$ Associate Professor, Division of Pediatric Dentistry, Department of Human Development and Fostering, Meikai University School of Dentistry, Japan

${ }^{2}$ Professor, Division of Pediatric Dentistry, Department of Human Development and Fostering, Meikai University School of Dentistry, Japan

${ }^{3}$ Professor, Division of Developmental Stomatognathic Function Science, Department of Health Promotion, Kyushu Dental University, Japan

\section{Oral topical distribution of some trace elements could be related to caries prevalence}

\section{What is trace elements?}

Trace elements are the elements that are essential in life action. Each trace element is maintained in living organisms at the very low level. They also distribute in nature surroundings such as earth crust.

The author and his colleagues investigated concentration of some trace elements in resting mixed saliva of children [1,2]. Some interesting findings will be shown briefly.

\section{Trace elements in resting mixed saliva}

Collection of resting mixed saliva and measurement of trace elements: The author and his colleagues compared concentration of trace elements in resting mixed saliva among children based on their oral status $[1,2]$. In the previous studies, resting mixed saliva was collected in the following way;

\#1. Collection was carried out at 10:00 - 11:30 a.m.

\#2. Donor children rinsed his/her mouth three times with ultrapure water just before collection.

\#3. 5-10 mL of resting mixed saliva was collected in individual sterilized test tube.

\#4. The test tube was sealed and frozen.

Concentration of each trace element (Manganese, Copper, Aluminum, and Iron) in individual child was determined by simultaneous multi-element atomic absorption spectrometry with Zeeman background correction (model Z-9000, Hitachi, Tokyo, Japan).

Copper level in resting mixed saliva: It was founded that the Copper level in resting mixed saliva increased significantly when the donor had caries history. And that no significant difference was found in the Copper concentration in resting mixed saliva between children who had already treated all caries teeth and children who had no caries history (Table 1). Moreover, the authors found that the Copper level was proportional to the number of untreated carious teeth.

Aluminum level in resting mixed saliva: The author and his colleagues found that Aluminum level in resting mixed saliva decreased inversely with the number of deciduous teeth and increased with the number of permanent teeth. Topical application of Aluminum had been reported to be cariostatic $[3,4]$. The author and his colleagues discussed in their report that Aluminum eluted more from immature enamel than from enamel of deciduous teeth. There may be a protective system against dental caries in immature enamel of releasing Aluminum into oral fluid, which prevent plaque development.

Possibilities of adopting trace elements in resting mixed saliva as one of the parameters of individual caries proneness

The author and his colleagues have been working on measuring concentration of some trace elements in resting mixed saliva and evaluating relationship between their concentration and caries prevalence. Resting mixed saliva is one of the ideal specimen materials because it is non-invasive and easy to use. If some relationship is found between the level of some trace element and caries prevalence, the measurement of the trace element in resting mixed saliva will be ideal screening of individual caries proneness and contribute to making better prognosis of oral hygiene instruction.

\section{Evaluation of enamel mineral density using micro-CT}

\section{Concept of enamel mineral density}

Dentists take their patients' radiographs in regular oral checkups and sometimes find early demineralized lesion. In some cases, the dentists who find early demineralized lesion have to make a very difficult decision; whether they should drill the lesion or not? Generally speaking, the very early demineralized lesion within enamel could be

Correspondence to: Shigeru Watanabe, Department of Human Development \& Fostering, Division of Pediatric Dentistry, School of Dentistry, Meikai University, 1-1 Keyakidai, Sakado, Saitama 350-0283, Japan, Tel: (+81) 49-279-2743, Fax: (+81) 49-279-2743, E-mail: shigeru@dent.meikai.ac.jp

Special Issue: Early Caries (Surbsurface Demineralization)

Shigeru Watanabe, DDS, PhD

Professor of Division of Pediatric Dentistry,

School of Dentistry, Meikai University, Japan

Published: April 12, 2017 
remineralized so they usually perform remineralizing therapy such as topical application of fluoride and instructions on daily habit and teeth brushing. When the dentists provide these therapy and instructions, it is very important for them to know exactly how serious the demineralized lesion is; otherwise, they can't make a correct prognosis, and then, fail in providing persuasive instructions for their patients.

When doctors make a diagnosis of osteoporosis, they use "bone mineral density". It is determined as "a measure of the amount of minerals contained in a certain volume of bone". In order to ensure exact and objective evaluation on demineralized lesion, the author introduced the concept of mineral density in diagnosing enamel early demineralized lesion in enamel [5].

\section{Measurement of enamel mineral density using hounsfield unit value}

When X-ray travels through an object, it attenuates based on the density, atomic number, and thickness of the object, as well as wave length of the X-ray. When the object contains much minerals including calcium, the X-ray will be attenuated after traveling through the object, resulting in white gradient in the Computed Tomography (CT) image.

CT image consists of pixels or voxels. The gradient of each pixel or voxel is determined by CT value; Hounsfield Unit Value (HUV). The HUV varies according to the density (radiolucency) of the object, and is determined as 0 and -1000 when the $\mathrm{X}$-ray travels through water and air, respectively.

\section{Evaluation of enamel mineral density using micro-CT an in vitro study}

Preparation of enamel specimen and measurement of HUV: Labial surfaces of crown of 8 bovine incisors were covered with nail varnish to make 4 square windows measuring $1 \mathrm{~mm}^{2}$. The enamel specimens were incubated in lactic acid solution that contained $0.1 \mathrm{M}$ of lactic acid and $6 \mathrm{wt} \%$ of CM-cellulose ( $\mathrm{pH} 3.7)$ at $38^{\circ} \mathrm{C}$. Windows were covered one by one at $30,60,90$, and 120 minutes of incubation. HUV of each window was measured using SKYSCAN1172 (SKYSCAN, Kontich, Belgium) under the following conditions: selection of highresolution camera $(1,280 \times 1,024$ pixels), source voltage of $100 \mathrm{kV}$, source current of $100 \mu \mathrm{A}$, image pixel size of $18 \mu \mathrm{m}$, frame averaging of 3 , random movement of $10, \mathrm{Al}$ filter, rotation step of 0.3 degrees, 360 degree-rotation, flat field correction.

HUV based on the time of demineralization: The mean HUV was significantly higher in enamel without immersion in acid $(5,195 \mathrm{HU})$ than in enamel of the window area (30 minutes of incubation; 2,384 $\mathrm{HU}, 60$ minutes of incubation; 2,382 HU, 90 minutes of incubation; 2,319 $\mathrm{HU}$, and 120 minutes of incubation; 2,294 HU). In this way, HUV enables dentists to evaluate enamel mineral density objectively and helps them to make accurate diagnosis and exact prognosis of the lesion.

HUV based on the depth from the enamel surface: As mentioned before, micro-CT enables dentists to evaluate the time course changes in enamel mineral density objectively. Mineral distribution in enamel can be also measured based on the depth from the enamel surface at one time point using micro-CT. Figure 1 [5] shows the mean HUV based on the depth from the enamel surface in enamel specimen which were demineralized for 60 minutes. HUV began to decrease at the depth of $54 \mu \mathrm{m}$. The UHV increased once at the depth of $288 \mu \mathrm{m}$, and kept decreasing again till the depth reached around the enamel-dentin junction. The manuscript reported by the author at al. [5] suggested

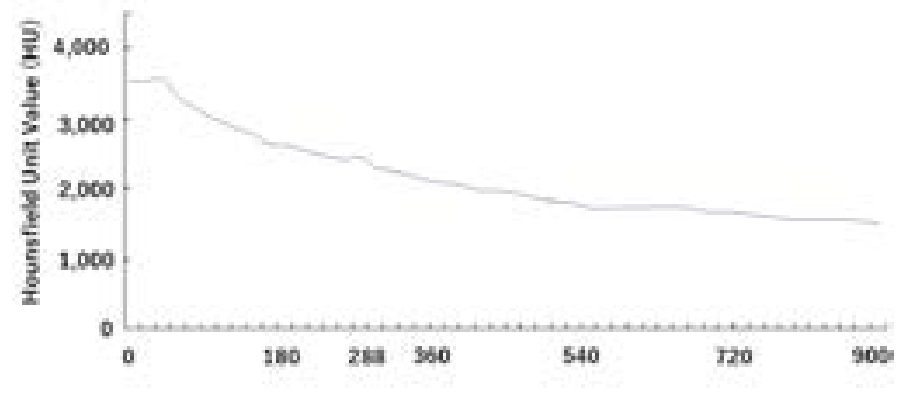

Figure 1. Depth from the enamel surface $(\mu \mathrm{m})$.

Table 1. Comparison of copper levels in resting mixed saliva of children based on oral status

\begin{tabular}{|c|c|c|c|c|}
\hline \multirow{2}{*}{ Oral Status } & \multirow{2}{*}{ Caries Histry (-) } & & \multicolumn{2}{|c|}{ Caries History (+) } \\
\cline { 3 - 5 } & & & Untreated Caries & Treated Caries \\
\hline Copper Level & $3.8 \mathrm{ng} / \mathrm{mL}$ & $\Leftrightarrow$ & Higher & NS \\
\hline
\end{tabular}

that the boundary of the very early demineralized region could be around a depth of $216<\mathrm{D} \leq 288 \mu \mathrm{m}$ in the enamel that was demineralized for 60 minutes, and there could be a high mineral distribution near the enamel surface and the boundary (bottom) of the very early and severely demineralized region. If dentists can diagnose enamel mineral density using CT, three-dimensional and objective evaluation on demineralized lesion will be possible, and then, appropriate control of the early demineralized lesion based on exact diagnosis and prognosis will be possible.

Agendas of evaluating early demineralized lesion using microCT: Micro-CT is designed for animal experiment, so if dentists want to introduce CT into evaluation on early demineralized lesion, they have to buy CT for human being that has similar (high) definition to micro-CT of SKYSCAN1172. When they take CT images in their dental office, they have to get the images under the same condition and settings; otherwise, it is impossible to compare HUVs between/ among time points during control of the lesion. Also, exposure should be diminished to get CT images every several months. If the technical and economic problems are solved, CT could be one of the options to evaluate early demineralized lesion.

\section{References}

1. Watanabe K, Tanaka T, Shigemi T, Hayashida Y, Maki K (2009). Mn and $\mathrm{Cu}$ concentrations in mixed saliva of elementary school children in relation to sex, age, and dental caries. J Trace Elem Med Biol 23: 93-99. [Crossref]

2. Watanabe K, Tanaka T, Shigemi T, Saeki K, Fujita Y, Morikawa K, et al. (2011). A and Fe levels in mixed saliva of children related to elution behavior from teeth and restorations. J Trace Elem Med Biol 25: 143-148.

3. Bihani SN, Damle SG (1997) Evaluation of an alum containing mouthrinse on plaque and gingivitis inhibition over 2 weeks of supervised use. J Indian Soc Pedod Prev Dent 15: 34-38. [Crossref]

4. Putt MS, Kleber CJ, Smith CE (1996) Evaluation of an alum-containing mouthrinse in children for plaque and gingivitis inhibition during 4 weeks of supervised use. Pediatr Dent 18: 139-144. [Crossref]

5. Watanabe K, Nakamura T, Ogihara T, Ochiai Y, Watanabe S (2012) Longitudina evaluation of mineral loss at the earliest stage of enamel demineralization using microcomputed tomography. Health 4: 334-340.

Copyright: (C2017 Watanabe K. This is an open-access article distributed under the terms of the Creative Commons Attribution License, which permits unrestricted use, distribution, and reproduction in any medium, provided the original author and source are credited. 